\title{
A Double-Blind, Placebo Controlled Clinical Trial Evaluating the Efficacy and Safety of a New Skin Whitening Combination in Patients with Chloasma
}

\author{
Xi Wang ${ }^{1,2}$, Zhaoxia Li², Dan Zhang2, Li Li ${ }^{1,2}$, Seite Sophie ${ }^{3^{*}}$ \\ ${ }^{1}$ Department of Dermatology, West China Hospital, Sichuan University, Chengdu, China \\ ${ }^{2}$ Sichuan Province Cosmetics Engineering Technology Research Centre, Chengdu, China \\ ${ }^{3}$ La Roche-Posay Pharmaceutical Laboratories, Asnieres, France \\ Email: sophie.seite@loreal.com
}

Received 3 December 2013; revised 3 January 2014; accepted 10 January 2014

Copyright (C) 2014 by authors and Scientific Research Publishing Inc.

This work is licensed under the Creative Commons Attribution International License (CC BY). http://creativecommons.org/licenses/by/4.0/

\section{Open Access}

\begin{abstract}
Melasma (or chloasma) is a hyperpigmentation disorder predominantly affecting sun-exposed skin in women, and is often refractory to treatment. The objective was to evaluate the efficacy and safety of a new whitening formula for the treatment of chloasma. This single centre, double-blind, placebo controlled, bilateral (split-face) comparison, was conducted on 44 Chinese subjects with chloasma during the winter season. The test formula was applied twice a day, for 12 weeks on left side of the face and a placebo formula on the right side of the face. Assessments included the use of the hemi-MASI (split-face Melasma Area Severity Index), both ultraviolet and standard photography, together with clinical evaluations of efficacy and safety at T0, T6 and T12 weeks. A significant difference between the 2-hemi-MASI was noticed after 6 and 12 weeks of treatment. This result was confirmed by the clinical evaluation of the dermatologists who recorded a significant improvement in the half-face treated with the new whitening formula compared with that treated with placebo $(p=0.003)$. The tolerance of the new formula was recorded as excellent by $82 \%$ of subjects and found to be cosmetically appealing. In this study, the new whitening formula containing ferulic acid, Ginkgo Biloba, lipohydroxyacid (LHA), niacinamide and thermal spring water was safe and significantly improved chloasma after a 3-month-treatment period compared with placebo.
\end{abstract}

\section{Keywords}

Chloasma; Melasma; Hemi-MASI; Skin-Whitening; Ferulic Acid; Ginkgo Biloba; LHA; Niacinamide;

*Corresponding author.

How to cite this paper: Wang, X., Li, Z.X., Zhang, D., Li, L. and Sophie, S. (2014) A Double-Blind, Placebo Controlled Clinical Trial Evaluating the Efficacy and Safety of a New Skin Whitening Combination in Patients with Chloasma. Journal of Cosmetics, Dermatological Sciences and Applications, 4, 92-98. http://dx.doi.org/10.4236/jcdsa.2014.42014 


\section{Introduction}

Chloasma, or melasma, is a pigmentary disorder that typically appears on the face, forehead, cheeks and chin. It occurs most frequently in women with Fitzpatrick skin type III or higher, especially those of Asian origin and is associated with negative psychological and emotional effects [1]-[7].

Chloasma can result from pregnancy or taking oral contraceptives, but it also occurs spontaneously. It can affect up to $50 \%$ - 70\% of pregnant women [5] [6], but genetic, ethnic (skin type), hormonal, and environmental factors, i.e. ultraviolet (UVA and UVB) exposures [6] [7], are also implicated. Its treatment is difficult and the effectiveness of topically applied depigmenting agents (containing hydroquinone) or chemical peels (Jessner's solution, glycolic acid, lactic acid, alphahydroxyacids, ascorbic acid, etc.) has been assessed [5] [7]-[10]. However, overall, the results are unsatisfactory, and sometimes side-effects are quite significant.

It is well established that tyrosinase is a key enzyme in the synthesis of melanin within the melanocyte. In the melanin synthetic pathway, it catalyzes the hydroxylation of tyrosine to 3,4-dihydroxyphenylalanine (DOPA) and the oxidation of DOPA to dopaquinone. Tyrosinase inhibitors are thus the active ingredients in most commercially available skin-lightening cosmetics. Ferulic acid and Ginkgo Biloba have been shown to inhibit the activity of tyrosinase [11] [12]. Furthermore, ferulic acid is a potent, widespread plant antioxidant, which has potentially several different pharmacological activities, capable of preventing the occurrence, and reducing the severity of UV-induced skin damage, skin aging and pigmentary disorders [13].

Lipohydroxyacid or LHA has an exfoliating action on the skin that is efficacious at low concentrations, in addition to its possible anti-inflammatory properties [14]. Niacinamide (or nicotinamide) is the amide of vitamin B3, an essential dietary vitamin. One study suggested that topical treatment with niacinamide may have some beneficial effects on cutaneous hyperpigmentation via suppression of melanosome transfer [15]. Lastly, La Roche-Posay thermal spring water has demonstrated a protective action against the short- and long-term deleterious effects of radical oxygen species induced by, among others, UV exposure (i.e. anti-oxidant, immunomodulating and anti-carcinogenic effects). La Roche-Posay thermal spring water has also been studied for its antiinflammatory and anti-irritating potential [16].

Therefore, the primary objective of this study is to assess the efficacy of a new whitening formula as a treatment for chloasma evaluated in a single-centre, placebo controlled, bilateral (split-face) comparative study. Secondary objectives are to assess this new formula on both local and general safety, tolerability and to evaluate the skin acceptability in Chinese patients with melasma.

\section{Materials and Methods}

\subsection{Study Design}

This double-blind, single-centre placebo controlled, bilateral (split-face) comparative study was undertaken during the winter of 2010-2011 in Chengdu, China. The study protocol was reviewed and approved by a local ethics committee. Informed consent and photography consent were obtained from each subject before enrollment.

\subsection{Subjects}

Forty four Chinese women, 25 to 45 years old with chloasma were invited to attend a baseline assessment during which a medical history was taken and a physical examination was carried out by a dermatologist. Patients were excluded from the study if they were pregnant or breastfeeding, if they were receiving hormone or corticosteroid therapy or if they had a history of endocrine disorders or allergies. The use of depigmenting or whitening products (oral or topical) within the previous 6 weeks was also a ground for exclusion.

Chloasma was characterized according to pattern (centrofacial, mandibular or malar), type (epidermal, dermal or mixed) determined using Wood's light, and overall severity, as assessed by the Melasma Area and Severity Index (MASI) [17]. 


\subsection{Test Materials}

The test formula contained ferulic acid, Ginkgo Biloba, lipohydroxyacid (LHA), niacinamide and La RochePosay thermal spring water in a vehicle base. A neutral cream was used as placebo.

\subsection{Test Procedure}

Both study formulas were identical in appearance and supplied with two identical pump dispensers. One test formula was applied twice a day on the subjects' right face and the other one on the left face. Products were applied by clinical staff from Monday to Friday and by subjects themselves on the week-ends. These applications were performed in the morning and evening (with an 8 hour break), for a period of 12 weeks. Throughout the trial period, all subjects were required to use the same face-washing products and prevented from using new face-care products. All patients were instructed to avoid sun exposure and to not use any sunscreen.

\subsection{Assessment Time Points and Evaluation Parameters}

The MASI index was used to assess the severity of melasma. Total MASI score has been previously described by Kimbrough-Green et al. [17]. For this study, a new hemi-MASI derivation was calculated to obtain a left and a right MASI for each hemi-face and evaluate both treatments. The Right-MASI corresponded to 0.15 (D + H) A Forehead $+0.3(\mathrm{D}+\mathrm{H})$ A Right malar $+0.05(\mathrm{D}+\mathrm{H})$ A Chin and the Left-MASI to $0.15(\mathrm{D}+\mathrm{H})$ A Forehead $+0.3(\mathrm{D}+\mathrm{H})$ A Left malar + $0.05(\mathrm{D}+\mathrm{H})$ A Chin and varied from 0 to 24 .

At each visit, changes in skin colour were measured by a spectrophotometer (CM-2600d, Minolta, Japan). Melanin index and erythema index were evaluated by reflectance measurements using a Mexameter (MX 18; Courage \& Khazaka, Germany).

At baseline, 6 and 12 weeks, ultraviolet and standard antero-posterior, bilateral photographs at a $45^{\circ}$ angle were taken with a professional photographic system (VISIA-CR, Canfield, USA).

Dermatologists performed clinical evaluations at each time point. Both dermatologists and subjects evaluated the erythema, scaling, itching, burning of each hemi-face using a four-degree scale (none, mild, moderate and severe) in order to assess tolerance. Cosmetic acceptance was determined via patient questionnaire.

\subsection{Statistical Analysis}

Data analysis was performed with SPSS 13.0. The split-face design provides a consistent baseline allowing repeated measurements and analysis, enabling each subject to be his own control. Paired t-tests were conducted on normally distributed data. Otherwise, the non-parametric Wilcoxon Rank test was used. $\mathrm{p}<0.05$ was considered to be statistically significant.

\section{Results}

\subsection{Study Population}

A total of 44 Chinese women aged 25 - 45 years; skin type III and IV, all with an epidermal chloasma, 39 of which, exhibited a malar pattern, completed the study. The characteristics of the study participants are described in Table 1 and their sun-exposure and sun-protection habits in Table 2. More than $40 \%$ of subjects had never used any sunprotection and $73 \%$ already used whitening/lightening products.

The average MASI of patients at baseline was $15.8 \pm 6.9$ (from 3 to 34) and 26 patients (59\%) had a MASI between 10 and 30. There was no difference between the hemi-MASI distribution between treatment groups (7.8 \pm 3.64 versus $7.95 \pm 3.52$ for each hemi-MASI respectively) $(\mathrm{p}=0.595)$.

\subsection{Efficacy Analysis}

Irrespective of treatment (test formula or placebo), a significant reduction of both hemi-MASI was observed over time (Figure 1). Furthermore, a significant difference between each hemi-MASI was noticed after 6 weeks $(\mathrm{p}<0.05)$, which became highly significant after 12 weeks of treatment (Figure 1).

The changes in both hemi-MASI throughout the study are shown in Table 3, and Figure 2 illustrates the 
Table 1. Patient demographics.

\begin{tabular}{|c|c|}
\hline Criteria & Number of patients $(\%)(\mathrm{N}=44)$ \\
\hline Age $^{*}$ (years) & $41 \pm 4$ \\
\hline $\begin{array}{l}\text { Fitzpatrick skin type: } \\
-\quad \text { III } \\
-\quad \text { IV }\end{array}$ & $\begin{array}{l}20(45 \%) \\
24(55 \%)\end{array}$ \\
\hline Duration of chloasma* (years) & $7 \pm 5(1-20)$ \\
\hline $\begin{array}{ll}\text { Chloasma related to: } \\
- & \text { Pregnancy } \\
- & \text { Hormonal therapy } \\
- & \text { Sun-exposure } \\
- & \text { Cosmetic use } \\
- & \text { Other }\end{array}$ & $\begin{array}{c}17(39 \%) \\
0 \\
30(68 \%) \\
7(16 \%) \\
16(36 \%)\end{array}$ \\
\hline Number of pregnancies ${ }^{*}$ & $2 \pm 1(1-4)$ \\
\hline Age of the first pregnancy* (years) & $24 \pm 4$ \\
\hline Smoker & $2(5 \%)$ \\
\hline
\end{tabular}

" Data are mean \pm SD (Min-Max).

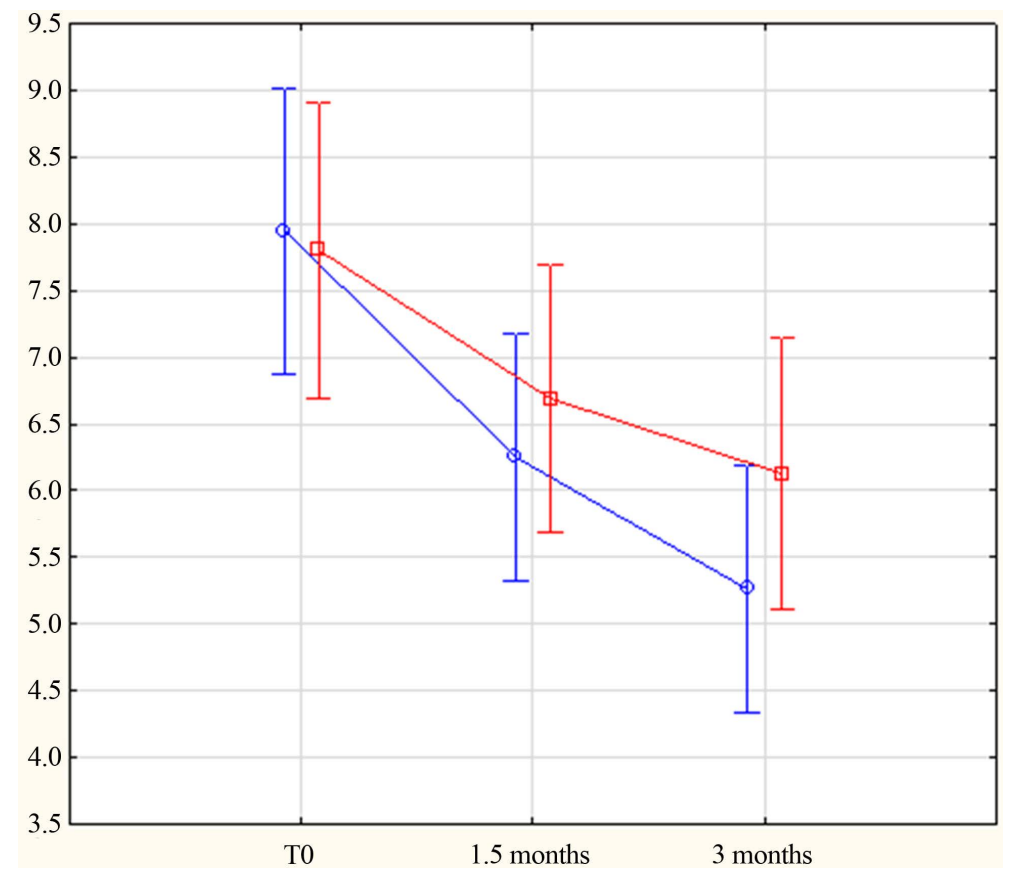

Figure 1. Changes of both hemi-MASI over time as a function of time. HemiMASI 95\% confidence intervals evolution over time differences between both treatments are significant $\left({ }^{*} \mathrm{p}<0.05\right)$ at 6 weeks, and highly significant ${ }^{* * *} \mathrm{p}<$ 0.001 ) at 12 weeks. Red line corresponds to placebo formula and blue line to test formula.

clinical results observed.

Neither colorimetric measurement $\left(\mathrm{L}^{*}, \mathrm{a}^{*}\right.$ and $\mathrm{b}^{*}$ values) nor Melanin Index obtained over time (chin, malar and forehead areas) of each hemi-face showed any statistical difference between the 2 treatments (data not shown).

\subsection{Safety and Global Efficacy Assessments}

A slight tingling sensation, which disappeared after continued use, was reported in $5 \%$ of subjects whatever their 
Table 2. Sun-exposure and sun-protection habits of patients.

\begin{tabular}{lc}
\hline Criteria & Number of patients (\%) (N = 44) \\
\hline Number of hours spent outside for work $^{*}$ & $0.7 \pm 1.5(0-8)$ \\
Number of hours spent outside for leisure* & $1.2 \pm 0.9(0-4)$ \\
Number of hours spent outside for vacation & \\
& $2 \pm 1.5(0-6)$ \\
Sun-protection: & \\
$-\quad$ Never & $19(43 \%)$ \\
$-\quad$ Sometimes & $15(34 \%)$ \\
$-\quad$ Often & $7(16 \%)$ \\
SPF (N = 25): & $3(7 \%)$ \\
$-\quad 15-20$ & \\
$-\quad 25-30$ & $6(24 \%)$ \\
$-\quad$ Unknown & $15(60 \%)$ \\
UVA-PF (N $=25):$ & $4(16 \%)$ \\
$-\quad$ PA+ & \\
$-\quad$ PA++ & $5(20 \%)$ \\
$-\quad$ PA+++ & $6(24 \%)$ \\
$-\quad$ Unknown & $5(20 \%)$ \\
Use of lightening product & $9(36 \%)$ \\
\hline
\end{tabular}

${ }^{*}$ Data are mean \pm SD (Min-Max).

Table 3. Changes of both hemi-MASI throughout the study.

\begin{tabular}{cccc}
\hline Results (Delta vs. baseline) & Test formula & Placebo & $\mathrm{P}^{*}$ \\
\hline 6-week treatment & $-1.69 \pm 1.32$ & $-1.12 \pm 1.10$ & 0.0024 \\
12-week treatment & $-2.68 \pm 1.74$ & $-1.68 \pm 1.50$ & 0.0002 \\
\hline
\end{tabular}

Mean \pm SD, ${ }^{*}$ Paired t-test p value.
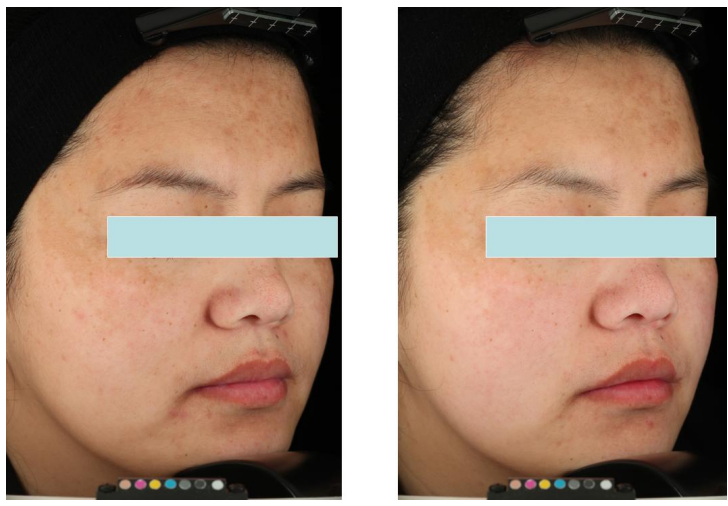

(a)
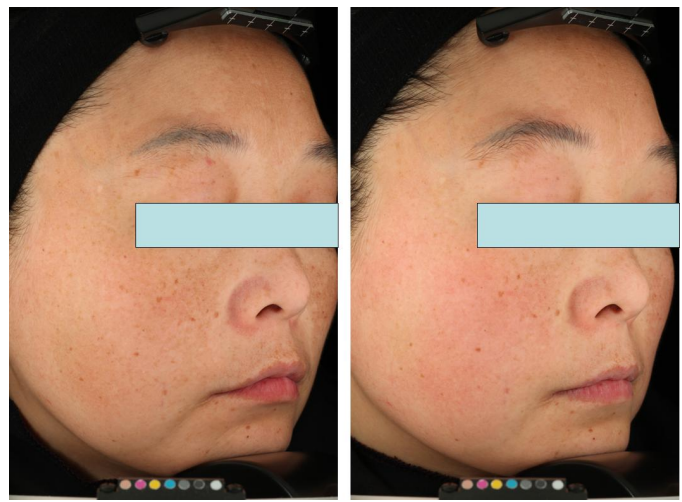

(b)

Figure 2. Clinical aspect of subjects before treatment and after the 12-week treatment. (a) 28-year-old woman; (b) 45-year-old woman.

treatment. No discomfort, topical or systemic adverse reactions were reported throughout the study. Excellent tolerance was experienced by $82 \%$ of patients for both formulas and there was no difference between the 2 treatments.

The efficacy was assessed by the study investigators as "good" in 32\% of cases on the hemi-face treated with the test formula and for only $9 \%$ on the hemi-face treated with the placebo formula $(\mathrm{p}=0.003)$.

The cosmetic properties (i.e. texture, smell, penetration, smoothness, radiance and moisturizing effect) of the 
test formula were more accepted, by the patients, than for the placebo formula.

\section{Discussion}

In this split-face study, the new whitening formula evaluated was shown by both clinical and objective assessments to produce significant improvements in chloasma pigmentation after 12 weeks of treatment compared with a placebo formula. For this study we developed and used a new hemi-MASI score (or split-face Melasma/ chloasma Area Severity Index), taking into account Area (A), Darkness (D) and Homogeneity (H), 3 key parameters of this pigmentation disorder. This method appeared to demonstrate and highlight sensitivities of the lightening/whitening effects of the active, test formula.

Ferulic acid, also named 4-hydroxy-methoxy cinnamic acid, is present in many traditional Chinese medicines such as Chuanxiong, horsetails, Cimicifuga and others [18]. Recently, it has received increasing attention in the cosmetics field, due to its efficacy in whitening, freckling, and as a sunscreen, amongst other purposes. It has been reported to absorb the ultraviolet rays between 290 and $330 \mathrm{~nm}$ [19] and can inhibit or reduce the proliferative activity of melanocytes and the activity of tyrosinase at the same time [20] [21]. In order to increase the skin-lightening activity of ferulic acid, it may be combined with other extracts, which have a different activity (for example Ginkgo Biloba, niacinamide and thermal spring water). The formulation may be further optimized by adding a keratolytic agent such as LHA [14].

Through a self-controlled study of the right and left face, we can determine whether this association of active ingredients can be applied, as a whitening agent, to improve and prevent facial hyperpigmentation. In this study, the efficacy and the tolerance of this formula were evaluated in the treatment of epidermal chloasma in Chinese women utilizing a bilateral (split-face) model. The clinical evaluation of chloasma using MASI (validated and widely used) showed that the test formula can significantly improve the clinical state of epidermal chloasma, after 6 weeks of application, and this improvement increased after 12 weeks of treatment. Self-assessment and photographs demonstrated the same result and were consistent with the dermatologist's evaluation.

In this study, colorimetric evaluation did not reveal any differences between the two treatment sides. The uneven colour of chloasma may have contributed to this finding, as subtle differences in the test values may not be accurately reflected in the treatment outcome. Further improvement in the study methodology is required in order to reduce any coincidence at each test site. The instrument test probes used in the study may have been too small and a pigment analyser with larger probes may provide more accurate data for uneven pigmentation diseases.

At various time points, the Melanin Index evaluated on the chin indicated a statistical difference in melanin contrast between the two sides, unlike the forehead and cheeks where there was no significant difference between the sides.

\section{Conclusion}

Nevertheless, these results suggest that a formula containing ferulic acid, Ginkgo Biloba, lipohydroxyacid (LHA), niacinamide and thermal spring water could effectively improve pigmentation in patients with epidermal melasma.

\section{Acknowledgements}

Editorial support was provided by Speak the Speech Consulting (Paris, France).

\section{Funding}

This study was funded by La Roche-Posay Pharmaceutical Laboratories, France.

\section{Conflict of Interest Statement}

S. Seité is employee of La Roche-Posay Pharmaceutical Laboratories, France.

\section{References}

[1] Jimbow, M. and Jimbow, K. (1989) Pigmentary Disorders in Oriental Skin. Clinics in Dermatology, 7, 11-27. 
http://dx.doi.org/10.1016/0738-081X(89)90053-9

[2] Chung, J.H. (2003) Photoaging in Asians. Photodermatology, Photoimmunology \& Photomedicine, 19, 109-121. http://dx.doi.org/10.1034/j.1600-0781.2003.00027.x

[3] Ho, S.G. and Chan, H.H. (2009) The Asian Dermatologic Patient: Review of Common Pigmentary Disorders and Cutaneous Diseases. American Journal of Clinical Dermatology, 10, 153-168. http://dx.doi.org/10.2165/00128071-200910030-00002

[4] Wand, R., Xu, Z., Tang, Y., Zhang, J., Wang, Z., Cao, L., Shen, Z., Zhou, C., Ding, X., Zheng, D., Gui, B. and Zhu, X. (2010) Prevalence of Melasma in Chinese Han and Chinese Yi: A Survey in Liangshan Distric. The Chinese Journal of Dermatovenereology, 6, 546-548.

[5] Balkrishnan, R., Kelly, A.P., McMichael, A. and Torok, H. (2004) Improved Quality of Life with Effective Treatment of Facial Melasma: The Pigment Trial. Journal of Drugs in Dermatology, 3, 377-381.

[6] Perez, M.L. (2005) The Stepwise Approach to the Treatment of Melasma. Cutis, 75, 217-222.

[7] Victor, F.C., Gelber, J. and Rao, B. (2004) Melasma: A Review. Journal of Cutaneous Medicine and Surgery, 8, 97102. http://dx.doi.org/10.1007/s10227-004-0158-9

[8] Sharquie, K.E., Al-Tirkreety, M.M. and Al-Mahhadani, S.A. (2005) Lactic Acid as a New Therapeutic Peeling Agent in Melasma. Dermatologic Surgery, 31, 149-154. http://dx.doi.org/10.1111/j.1524-4725.2005.31035

[9] Espinal-Perez, L.E., Moncada, B. and Castanedo-Cazares, J.P. (2004) A Double Blind Randomized Trial of 5\% Ascorbic Acid vs. 4\% Hydroquinone in Melasma. International Journal of Dermatology, 43, 604-607. http://dx.doi.org/10.1111/j.1365-4632.2004.02134.x

[10] Torok, H.M., Jones, T., Rich, P., Smith, S. and Tschen, E. (2005) Hydroquinone 4\%, Tretinoin 0.05\%, Flucinoloneacetonide 0.01\%: A Safe and Efficious 12-Month Treatment for Melasma. Cutis, 75, 57-62.

[11] Lee, H.S. (2002) Tyrosinase Inhibitors of Pulsatillacernua Root-Derived Materials. Journal of Agricultural and Food Chemistry, 50, 1400-1403. http://dx.doi.org/10.1021/jf011230f

[12] Fong, P. and Tong, H.H. (2012) Insilico Prediction of the Cosmetic Whitening Effects of Naturally Occurring Lead Compounds. Natural Product Communications, 7, 1287-1294.

[13] Tsuno, T. and Kadota, M. (2002) Application to the Cosmetics as an Ultraviolet Ray Absorbent of Ferulic Acid. Fragrance Journal, 30, 137.

[14] Saint-Leger, D., Leveque, J.L. and Verschoore, M. (2007) The Use of Hydroxy Acids on the Skin: Characteristics of C8-Lipohydroxy Acid. Journal of Cosmetic Dermatology, 6, 59-65. http://dx.doi.org/10.1111/j.1473-2165.2007.00296.x

[15] Hakozaki, T., Minwalla, L., Zhuang, J., et al. (2002) The Effect of Niacinamide on Reducing Cutaneous Pigmentation and Suppression of Melanosome Transfer. British Journal of Dermatology, 147, 20-31. http://dx.doi.org/10.1046/j.1365-2133.2002.04834.x

[16] Seité, S. (2013) Thermal Waters as Cosmeceuticals: La Roche Posay Thermal Spring Water Example. Clinical, Cosmetic and Investigational Dermatology, 6, 1-6.

[17] Kimbrough-Green, C.K., et al. (1994) Topical Retinoic Acid (Tretinoin) for Melasma in Black Patients. Archives of Dermatology, 130, 727-733. http://dx.doi.org/10.1001/archderm.1994.01690060057005

[18] Xiang, D.K. (2003) Natural Plant Actives-Ferulic Acid. China Cosmetics (Industry), 20, 76.

[19] Staniforth, V., Huang, W.C. and Aravindaram, K. (2011) Ferulic Acid, a Phenolic Phytochemical, Inhibits UVB-Induced Matrix Metalloproteinases in Mouse Skin via Posttranslational Mechanisms. The Journal of Nutritional Biochemistry, 23, 443-451.

[20] Oresajo, C., Stephens, T., Hino, P.D., et al. (2008) Protective Effects of a Topical Antioxidant Mixture Containing Vitamin C, Ferulic Acid, and Phloretin against Ultraviolet-Induced Photodamage in Human Skin. Journal of Cosmetic Dermatology, 7, 290-297. http://dx.doi.org/10.1111/j.1473-2165.2008.00408.x

[21] Murray, J.C., Burch, J.A., Streilein, R.D., Iannacchione, M.A., Hall, R.P. and Pinnell, S.R. (2008) A Topical Antioxidant Solution Containing Vitamins C and E Stabilized by Ferulic Acid Provides Protection for Human Skin against Damage Caused by Ultraviolet Irradiation. Journal of the American Academy of Dermatology, 59, 418-425.

http://dx.doi.org/10.1016/j.jaad.2008.05.004 